\title{
Bovine colostrum is superior to enriched formulas in stimulating intestinal function and necrotising enterocolitis resistance in preterm pigs
}

\author{
Hanne K. Møller ${ }^{1,2}$, Thomas Thymann ${ }^{3}$, Lisbeth N. Fink ${ }^{1}$, Hanne Frokiaer ${ }^{2}$, Anne S. Kvistgaard ${ }^{4}$ \\ and Per T. Sangild ${ }^{3 *}$ \\ ${ }^{1}$ Department of Systems Biology, Technical University of Denmark, DK-2800 Lyngby, Denmark \\ ${ }^{2}$ Department of Basic Sciences and Environment, University of Copenhagen, DK-1871 Frederiksberg C, Denmark \\ ${ }^{3}$ Department of Human Nutrition, University of Copenhagen, DK-1958 Frederiksberg C, Denmark \\ ${ }^{4}$ Arla Foods Ingredients, DK-8260 Viby, Denmark \\ (Received 10 February 2010 - Revised 12 June 2010 - Accepted 16 July 2010 - First published online 20 August 2010)
}

\begin{abstract}
Milk contains immunomodulatory compounds that may be important to protect the immature intestine in preterm neonates from harmful inflammatory reactions involved in disorders like necrotising enterocolitis (NEC). We hypothesised that bovine colostrum and milk formulas enriched with sialic acids (SL), gangliosides (Gang) or osteopontin (OPN) would improve gastrointestinal function and NEC resistance in preterm neonates. Forty-seven caesarean-delivered preterm pigs were given total parenteral nutrition for $2 \mathrm{~d}$ followed by $1.5 \mathrm{~d}$ of enteral feeding. In Expt 1, a control formula was compared with an OPN-enriched formula ( $n$ 13), while Expt 2 compared a control formula with bovine colostrum or formulas enriched with Gang or SL ( $n$ 4-6). OPN enrichment decreased NEC severity relative to control formula $(P<0 \cdot 01)$, without any significant effects on NEC incidence, digestive enzyme activities and hexose absorption. Neither SL- nor Gang-enriched formulas improved NEC resistance or digestive functions, while all the intestinal functional parameters were significantly improved in pigs fed bovine colostrum, relative to formula. The effects in vivo were supported in vitro by bacteria- and dose-dependent modulation by colostrum whey of the cytokine response from bacteria-stimulated murine bone marrow-derived dendritic cells (DC). In conclusion, OPN had only moderate NEC-protective effects, while formulas enriched with Gang or SL were ineffective. The observed modulation of DC cytokine response by bovine colostrum whey in vitro may be due to a synergistic action of various milk bioactives, and it may explain its beneficial effects on NEC development and intestinal function in a piglet model of preterm infants.
\end{abstract}

Key words: Colostrum: Milk bioactives: Immunomodulation: Necrotising enterocolitis

Milk contains numerous bioactive substances including immunoglobulins, cytokines, growth factors and components possessing antibiotic and prebiotic activity ${ }^{(1,2)}$. Little is known about the biological effects of individual milk bioactives, despite the fact that natural milk improves intestinal function and development of a stable and beneficial microbiota in the gastrointestinal $\operatorname{tract}^{(3)}$ relative to milk formula. The anti-microbial and anti-inflammatory properties of milk are most pronounced for the first milk (colostrum), although most known milk bioactives can be found throughout lactation. Milk components with preliminary evidence of positive effects on the intestinal immune system and microbiota include osteopontin (OPN), gangliosides (Gang) and sialic acids (SL) $)^{(1,4-10)}$. Characterisation of the biological effect of such components is important for optimal production of infant milk formulas to be used when mother's milk is not available. Furthermore, these compounds could be particularly important for nutrition of sensitive newborns, such as preterm infants.

OPN is a phosphorylated acidic glycoprotein expressed by a number of different immune and non-immune cells and tissues, and it is also known as early T cell activation 1 protein $^{(4,11,12)}$. Secreted OPN has cytokine-like properties. It enhances interferon- $\boldsymbol{\gamma}$-producing $\mathrm{T}$ helper 1 cell responses by inducing production of IL-12 and suppressing production of IL-10 $0^{(4,13,14)}$. Hence, expression of OPN has been shown to be important in the efficient development of the $\mathrm{T}$ helper 1 immune response, and OPN is recognised as a key cytokine involved in T helper 1 cytokine expression at sites of inflammation. Many studies have reported increased production of OPN during various inflammatory processes ${ }^{(4,13-16)}$. Overall, OPN can be categorised as an important regulator of processes involved in inflammation and tissue repair; in particular, those responses characterised by the presence

Abbreviation: DC, dendritic cells; Gang, gangliosides; NEC, necrotising enterocolitis; OPN, osteopontin; PN, parenteral nutrition; SI, small intestine; SL, sialic acids; TPN, total PN.

*Corresponding author: Professor P. T. Sangild, fax +45353324 69, email psa@life.ku.dk 
of macrophages and T cells. Besides lymphocyte activation and involvement in a number of other steps of cell-mediated immunity, OPN is also involved in cell survival and wound repair ${ }^{(11,14)}$. Due to the multifunctional immunoregulatory cytokine properties of $\mathrm{OPN}$, and since it is present in such high amounts especially during early lactation, it is very likely that OPN plays a significant role in the early immunological development of infants.

Gang are complex glycosphingolipids comprising a hydrophobic ceramide tail attached to a hydrophilic oligosaccharide with at least one SL molecule. They are widely distributed in vertebrate tissues, attached to cell surfaces or secreted to plasma and other body fluids ${ }^{(17-20)}$. Breast milk contains Gang, and milk from different species has specific Gang profiles with contents varying during lactation $^{(18,19,21-23)}$. Certain glycosylated milk Gang may serve as false receptor analogues for bacterial adhesins. Thus, they may be able to modify the intestinal microbiota in the neonate ${ }^{(7,19)}$. In vitro, Gang exhibit immunosuppressive effects on a number of immunocompetent cells, and these effects are dependent on Gang structure, concentration and the type of immune cell affected ${ }^{(17,18,24)}$.

SL comprise a diverse group of acidic sugars with a nine-carbon backbone. Typically, these are found on the terminal ends of glycan chains attached to lipids and proteins covering surfaces of all cells and a variety of secreted proteins in vertebrates. Here, they serve as components of binding sites for pathogens and toxins that recognise the SL unit in combination with different underlying carbohydrate chains of the cell surface glycans ${ }^{(10,25)}$. SL moieties on, for example, Gang can therefore inhibit attachment of pathogenic bacteria species such as Vibrio cholerae and enterotoxic Escherichia coli by acting as decoy receptors ${ }^{(26)}$.

Dendritic cells (DC) reside in the Peyer's patches and lamina propria of the intestine and are important antigenpresenting cells of the gut-associated immune system. We have previously shown that murine bone marrow-derived DC respond strongly to bacterial stimuli ${ }^{(27)}$, and this response can be modulated with various milk components ${ }^{(18,28)}$. Hence, the DC assay can be used as a screening tool to study the immunomodulatory effects of various milk bioactives.

In preterm pigs, maternal colostrum protects against necrotising enterocolitis (NEC) in the small intestine $(\mathrm{SI})^{(29-31)}$, while infant formula leads to digestive and immune dysfunction and NEC lesions. We hypothesised that these diet differences are explained partly by the lack of milk bioactives like OPN, Gang and SL in commercial milk formulas, relative to mother's milk, or a diet like bovine colostrum. We therefore tested the in vivo intestinal response to milk formulas enriched with OPN, Gang or SL in preterm pigs. Furthermore, the immunomodulatory activity of the enriched milk fractions was investigated in vitro using DC stimulated with gut-associated bacteria. Both the overall bacterial load and specific groups (such as Clostridia) are suspected to play a role in NEC aetiology ${ }^{(32)}$.

\section{Materials and methods}

\section{Animals, surgery and nutrition protocols}

Forty-seven piglets (Large White $\times$ Danish Landrace) from three litters were delivered by caesarean section at $105 \mathrm{~d}$ of gestation as previously described ${ }^{(30)}$. While still anaesthetised from delivery, each piglet was fitted with an orogastric feeding tube (infant feeding tube 6F; Portex, Kent, UK) and a vascular catheter (4F; Portex) in the dorsal aorta via the transected umbilical $\operatorname{cord}^{(29)}$. Within $6 \mathrm{~h}$ of delivery, all the piglets were administered total parenteral nutrition (TPN), continuously infused through the arterial catheter for $48 \mathrm{~h}$. Initially, the rate was $4 \mathrm{ml} / \mathrm{kg}$ body weight per $\mathrm{h}$ advancing to $6 \mathrm{ml} / \mathrm{kg}$ body weight per $\mathrm{h}$ after $12 \mathrm{~h}$. The TPN solution was prepared aseptically as previously described and adjusted to meet the nutritional requirements of the preterm pig $^{(29,33,34)}$. All the pigs were immunised with three gradually increasing doses of porcine plasma obtained from their mother ${ }^{(35)}$.

Enteral milk formulas used as control diets were prepared from commercially available formula products (Pepdite, Maxipro, Liquigen; SHS International, Liverpool, UK) and adjusted to match the energy and protein concentrations in porcine milk during lactation. The three enriched milk formulas were prepared by the addition of pure bovine OPN or Gang- or SL-enriched bovine milk fractions (all from Arla Foods Ingredients, Viby J, Denmark) as described later. The protein, lipid and carbohydrate contents were adjusted according to the additional nutrients added to each formula to obtain similar compositions for all formulas within the same experiment. As the Gang- and SL-enriched milk products were not completely pure, like the OPN product, these were tested in a separate experiment where the control formula was adjusted to match the composition of the Gang and SL formulas. Hence, the formula control diets of the two experiments were slightly different. The final composition of the applied formulas is shown in Table 1. Bovine colostrum was collected and pooled from two to three Holstein cows from the first milking after calving and stored at $-20^{\circ} \mathrm{C}$ until use. The content of OPN, SL and Gang in bovine milk has been documented elsewhere ${ }^{(21,22,36)}$

After the TPN period, the pigs were switched to enteral nutrition. Every $3 \mathrm{~h}$, all the pigs were manually given oral boluses of $15 \mathrm{ml} / \mathrm{kg}$ of their respective diets, and the pigs were monitored every $3 \mathrm{~h}$ for potential clinical signs of NEC (feeding intolerance, abdominal distension, diarrhoea and respiratory stress). The pigs were euthanised for sample collection $1.5 \mathrm{~d}$ after initiation of enteral feeding unless serious clinical NEC symptoms developed beforehand. All animal protocols and procedures were approved by the Danish National Committee on Animal Experimentation.

\section{Expt 1: osteopontin-enriched formula to preterm pigs}

Twenty-six preterm pigs derived from three litters were randomly assigned to two treatment groups. During the 
Table 1. Composition of enriched (osteopontin (OPN), gangliosides (Gang) and sialic acids (SL)) and control formulas (per litre formula)

\begin{tabular}{|c|c|c|c|c|c|}
\hline & OPN & Control & Gang & SL & Control \\
\hline Energy $(\mathrm{kJ})$ & 4099.89 & 4051.85 & $3927 \cdot 32$ & 4171.20 & 3982.91 \\
\hline Protein $(\mathrm{g})$ & 61.77 & 63.20 & $58 \cdot 17$ & 63.63 & 60.52 \\
\hline OPN (g) & $2 \cdot 22$ & 0.00 & 0.00 & 0.00 & 0.00 \\
\hline Polycose (g) & 44.88 & 44.25 & $42 \cdot 64$ & $47 \cdot 20$ & $42 \cdot 64$ \\
\hline Dextrose $(\mathrm{g})$ & 8.40 & 8.78 & 3.85 & 4.00 & 3.85 \\
\hline Lactose $(\mathrm{g})$ & 0.02 & 0.00 & $8 \cdot 16$ & 10.56 & 8.80 \\
\hline Sialyl lactose (g) & 0.00 & 0.00 & 0.00 & 8.66 & 0.00 \\
\hline Gang (g) & 0.00 & 0.00 & 0.06 & 0.00 & 0.00 \\
\hline Protein-bound carbohydrate (g) & 0.24 & 0.00 & 0.00 & 0.00 & 0.00 \\
\hline Total fat $(\mathrm{g})$ & 62.29 & 60.15 & 56.45 & 57.45 & $56 \cdot 64$ \\
\hline Saturated fat $(\mathrm{g})$ & 46.06 & 43.94 & 38.79 & 41.36 & $41 \cdot 15$ \\
\hline Monounsaturated fat $(\mathrm{g})$ & 9.12 & $9 \cdot 21$ & 8.69 & 9.01 & 8.69 \\
\hline Polyunsaturated fat (g) & 3.43 & 3.44 & $3 \cdot 61$ & 3.74 & $3 \cdot 61$ \\
\hline
\end{tabular}

$2 \mathrm{~d}$ PN period, one group was given minimal enteral doses of $5 \mathrm{ml} / \mathrm{kg}$ body weight per $3 \mathrm{~h}$ of pure OPN diluted in sterile deionised water to a concentration of $2 \mathrm{~g} / \mathrm{l}$ (OPN, $n$ 13), while the controls ( $n$ 13) were given the same volume of deionised water. After the PN period, both the groups were switched to total enteral nutrition and fed either the standard milk formula (controls) or the same formula enriched with $2 \cdot 2 \mathrm{~g} / \mathrm{l}$ of OPN (OPN group).

\section{Expt 2: colostrum or gangliosides- or sialic acids-enriched} formulas to preterm pigs

Twenty-one pigs were obtained from three separate litters and administered TPN as described earlier. Following TPN, the pigs were assigned to a bovine colostrum group ( $n$ 5) and three formula groups. The formula groups were given either standard milk formula (control, $n$ 4), formula with an SL-enriched bovine milk fraction $(60 \mathrm{~g} / 1$ corresponding to $8.7 \mathrm{~g} / \mathrm{l}$ of sialyl lactose in the final solution, SL, $n$ 6) or formula with Gang-enriched bovine milk fraction ( $3 \mathrm{~g} / \mathrm{l}$, final concentration of Gang was $0.06 \mathrm{~g} / \mathrm{l}$ formula, Gang, $n$ 6). The number of animals allocated to the treatment groups in Expt 2 ( $n$ 4-6) was kept relatively small because the studies quickly documented high NEC severity scores in all three formula groups, with marked differences relative to bovine colostrum. The concentration of SL used in this experiment corresponded to the concentration in human colostrum $^{(7,37)}$, and immunomodulatory effects of milk proteins containing SL moieties have been documented in vitro and in vivo ${ }^{(9,25,28,37,38)}$.

\section{In vivo carbohydrate absorption}

The test was performed twice by the end of the PN period and $1 \mathrm{~d}$ after initiation of enteral nutrition. Boluses of $15 \mathrm{ml} / \mathrm{kg}$ body weight of $2 \%$ mannitol and $5 \%$ galactose were administered orally, and arterial blood samples were collected at intervals (0, 20, 40 and $60 \mathrm{~min})$ after the two boluses. Concentrations of mannitol and galactose in plasma were analysed spectrophotometrically as previously described ${ }^{(39,40)}$.

\section{Necrotising enterocolitis evaluation and tissue collection}

When clinical symptoms of NEC were observed in several pigs in a litter or no later than $1.5 \mathrm{~d}$ after initiation of enteral nutrition, the pigs were anaesthetised and subsequently killed with an intra-cardiac injection of sodium pentobarbitone $(60 \mathrm{mg} / \mathrm{kg})$. The entire gastrointestinal tract was removed, and the SI, colon and stomach were weighed without contents. SI length was measured in a relaxed state, and the intestine was divided into three equal segments designated proximal, middle and distal SI. The wet mass of lungs, liver, spleen, heart and kidneys was recorded, and the lung volume was determined by immersion in water. Samples from the three SI regions were snap frozen in liquid $\mathrm{N}_{2}$ and stored at $-80^{\circ} \mathrm{C}$ before analysis of enzymatic activity. Another set of samples was fixed in $4 \%$ neutral-buffered paraformaldehyde for villus morphology as described previously ${ }^{(29,33)}$.

Each SI segment, stomach and colon were photographed and given a macroscopic pathologic lesion score on a scale from 1 to 6 . Here, 1 indicates the absence of any NEC-associated symptoms; 2 indicates local hyperaemia, inflammation and oedema; 3 indicates hyperaemia, extensive oedema and local haemorrhage; 4 indicates extensive haemorrhage; 5 indicates local necrosis and pneumatosis intestinalis; and 6 indicates extensive necrosis and pneumatosis intestinalis. A pig having a score of 3 or more in any region was considered a case of NEC. An average NEC severity score across all five regions was calculated for each pig, and an average score for each treatment group was calculated from these average scores. The proportion of dry weight of mucosa was determined as previously described ${ }^{(30)}$.

\section{Intestinal mucosal morphology and enzyme activities}

Fixed SI samples were embedded in paraffin, sectioned $(3 \mu \mathrm{m})$, and mounted on glass slides and stained with haematoxylin and eosin. Villus heights of ten intact villi from one representative cross-section from each pig were measured on scanned images of the tissue sections using 
the software SoftWoRx Explorer version 1.1 (Applied Precision, Issaquah, Washington, DC, USA).

Homogenates of frozen samples from the three SI segments from each pig were assayed spectrophotometrically for activities of lactase, sucrase, maltase, dipeptidylpeptidase IV, aminopeptidase $\mathrm{N}$ and aminopeptidase A. Lactose, sucrose, maltose, glycyl-L-prolin-4-nitroanilide, L-alanine-4-nitroanilide and $\alpha$-L-glutamic acid 4-nitroanilide were used, respectively, as substrates, in accordance with a previously established protocol ${ }^{(41)}$. The hydrolytic release of $1 \mu \mathrm{mol}$ substrate per $\min$ at $37^{\circ} \mathrm{C}$ was considered to represent one unit (U) of enzyme activity, which was expressed per gram of wet intestine.

\section{Expt 3: in vitro immunomodulatory activity of milk bioactives}

Preparation of bacterial strains. Clostridium perfringens NEC A20 (type A isolate from a premature piglet with NEC) was grown anaerobically overnight at $37^{\circ} \mathrm{C}$ in tryptone soya broth (Oxoid A/S, Greve, Denmark). E. coli Nissle 1917 (O6:K5:H1, isolate from human faeces) was cultured aerobically in de Man, Rogosa and Sharpe broth (Merck, Darmstadt, Germany) overnight at $37^{\circ} \mathrm{C}$. Bacterial cultures were harvested by centrifugation at $2700 \mathrm{~g}$ for 15 min, washed twice in sterile PBS ( $\mathrm{pH} 7 \cdot 2)$ and resuspended in PBS. Bacterial cells were killed by $1 \mathrm{~h}$ exposure to UV light and then stored at $-80^{\circ} \mathrm{C}$. Bacterial
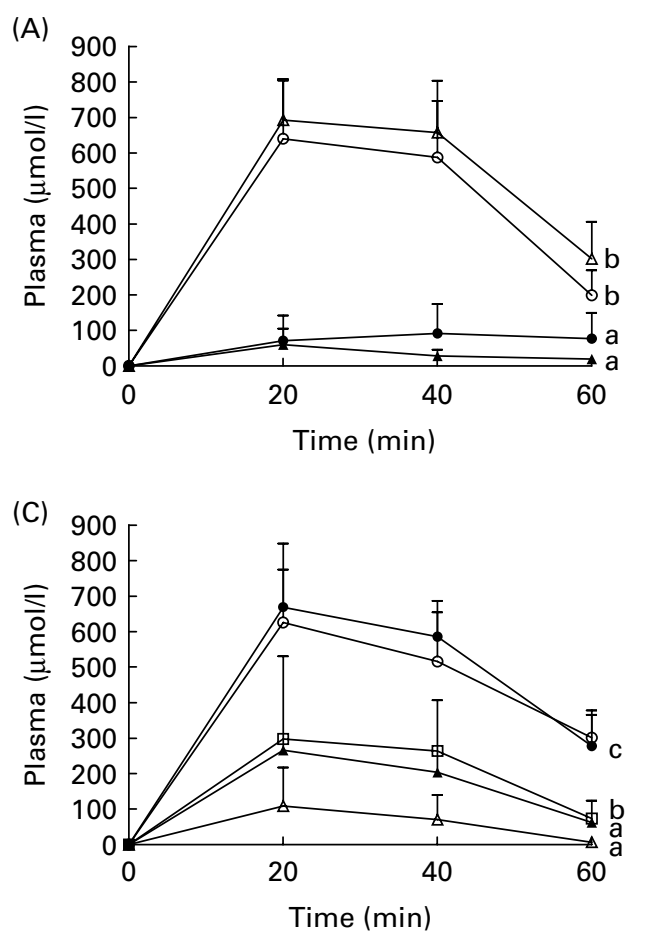

concentrations were determined from dry mass of freezedried samples and corrected for buffer salt concentration.

Preparation of dendritic cells and co-culturing with milk bioactives and bacteria. Bone marrow cells were flushed with PBS from the dissected femur and tibia from C57BL/6 mice. The cells were washed twice in PBS and resuspended in Roswell Park Memorial Institute-1640 supplemented with $2 \mathrm{~mm}$-L-glutamine, $10 \%$ (v/v) heat-inactivated foetal calf serum, $100 \mathrm{U} / \mathrm{ml}$ penicillin, $100 \mu \mathrm{g} / \mathrm{ml}$ streptomycin, $50 \mu \mathrm{m}$-2-mercaptoethanol (culture medium, all components from Lonza, Basel, Switzerland) and $15 \mathrm{ng} / \mathrm{ml}$ murine granulocyte-macrophage colony-stimulating factor (supernatant from a granulocyte-macrophage colony-stimulating factor transfected Ag8.653 myeloma cell line). The cells were incubated for $8 \mathrm{~d}$ in petri dishes at $37^{\circ} \mathrm{C}$ and $5 \% \mathrm{CO}_{2}$. On days 3 and 6 of incubation, the cells were supplied with fresh culture medium containing $15 \mathrm{ng} / \mathrm{ml}$ granulocyte-macrophage colony-stimulating factor. On day 8 , non-adherent cells were harvested and resuspended in culture medium with $10 \mathrm{ng} / \mathrm{ml}$ granulocyte-macrophage colony-stimulating factor. A total of $2 \times 10^{6}$ cells $/ \mathrm{ml}$ were seeded in forty-eight-well tissue culture plates, and $10 \mu \mathrm{g} / \mathrm{ml}$ bacteria were added together with different concentrations of OPN, SL, Gang, bovine colostrum or colostrum whey diluted appropriately in culture medium. Colostrum whey was produced by coagulation with $3 \mu \mathrm{l} / \mathrm{ml}$ bovine chymosin (Chr. Hansen, Horsholm, Denmark) for $30 \mathrm{~min}$ at $37^{\circ} \mathrm{C}$ and subsequent centrifugation for $10 \mathrm{~min}$ at $280 \mathrm{~g}$.
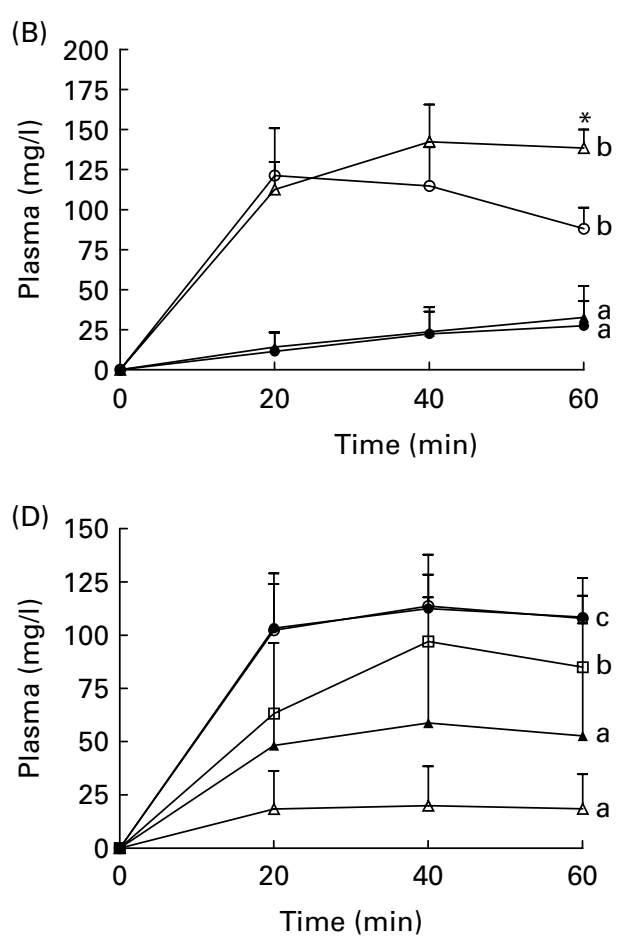

Fig. 1. Absorption of galactose $((A)$ and $(C))$ and mannitol $((B)$ and $(D))$ by the end of the $2 d$ total parenteral nutrition (TPN) period and $1 \mathrm{~d}$ after start of enteral feeding. Values represent increments in plasma galactose and mannitol at 20, 40 and 60 min (means with their standard errors). Enriched formulas are compared with colostrum and control formulas, respectively, and the entire response was analysed by repeated measures. ${ }^{a, b, c}$ Mean values with unlike superscript letters assigned to the curves indicate galactose and mannitol responses that differ significantly $(P<0 \cdot 05)$. ${ }^{*}$ Mean values for the TPN-osteopontin (OPN) and OPN groups differ significantly specifically at $60 \mathrm{~min}$ in $(\mathrm{B})(P<0.05$, by $t$ test). ((A) and $(\mathrm{C}))-\triangle-$, TPN-OPN; $-\mathrm{O}-$, TPN control; $-\mathbf{\Delta}-$, OPN; $-\bullet-$, control. ((B) and (D)) $-\bigcirc-$, TPN all pigs; $-\boldsymbol{\Delta}-$, sialic acids; $-\triangle-$, gangliosides; $-\bullet-$, colostrums; $-\square-$, control. 
The final volume in each well was $600 \mu$ l. The cells were stimulated for $18-20 \mathrm{~h}$ at $37^{\circ} \mathrm{C}$ and $5 \% \mathrm{CO}_{2}$ before collection of cell culture supernatants, which were stored at $-20^{\circ} \mathrm{C}$ until cytokine analysis. The concentration of cytokines IL-12p70, IL-10, IL-6 and TNF- $\alpha$ was determined using commercially available ELISA kits (R\&D Systems, Minneapolis, MN, USA) according to the manufacturer's instructions.

\section{Statistical analysis}

ELISA data from the DC experiments were evaluated by one-way ANOVA with Dunnett's multiple comparison post hoc test using Graph Pad Prism (version 5.01; Graph Pad Software Inc., La Jolla, CA, USA). Villi heights were compared with one-way ANOVA and Tukey's post boc test (Expt 2) or Student's $t$ test (Expt 1) using Graph Pad Prism. Enzyme activity and NEC severity score data were analysed by two-way ANOVA for the two in vivo experiments separately using the mixed procedure of Statistical Analysis Systems (SAS/STAT version 9.1; SAS Institute, Cary, NC, USA). Treatment and intestinal region were fixed effects, while pig and litter were regarded as random variables. Sugar absorption data were analysed with repeated-measures analysis using Statistical Analysis Systems. NEC incidence was analysed using Fisher's exact procedure test with subsequent pairwise comparisons of treatment groups. Data are presented as means with their standard errors or means and standard deviations in all statistical analyses; $P$ values $<0.05$ were considered significant.

\section{Results}

Expt 1 and 2: in vivo studies on enriched bovine milk fractions and colostrum

In vivo carbohydrate absorption. The in vivo response to oral boluses of galactose and mannitol by the end of the TPN period and $1 \mathrm{~d}$ after the initiation of enteral nutrition showed no significant difference between OPN and controls (repeated-measures analyses of graphs in Fig. 1). However, $60 \mathrm{~min}$ after the mannitol bolus, 2-d-old piglets supplemented with OPN during the TPN period had significantly elevated plasma mannitol levels, compared with controls $(P<0.05$, Fig. 1(B)). Both the groups had much lower plasma increments of galactose and mannitol during enteral feeding than during the TPN period.

In Expt 2 after initiation of enteral nutrition, Gang and SL lowered the galactose and mannitol absorption relative to controls, while the absorption capacity was higher in colostrum pigs compared with all formula-fed pigs (Fig. 1, $P<0 \cdot 01$ ).

Necrotising enterocolitis incidence and severity. OPN supplementation (Expt 1) did not reduce the incidence of NEC ( 54 and $77 \%$ in the OPN and control groups, respectively, Table 2). However, the mean NEC severity score

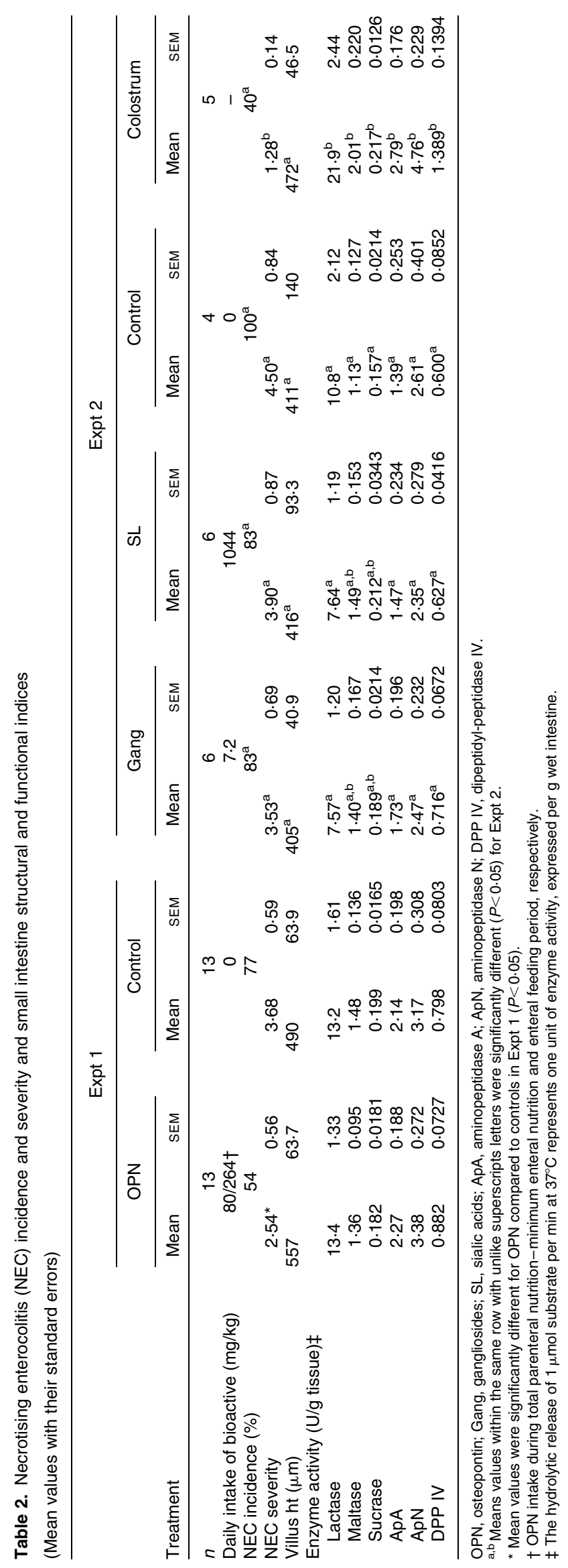




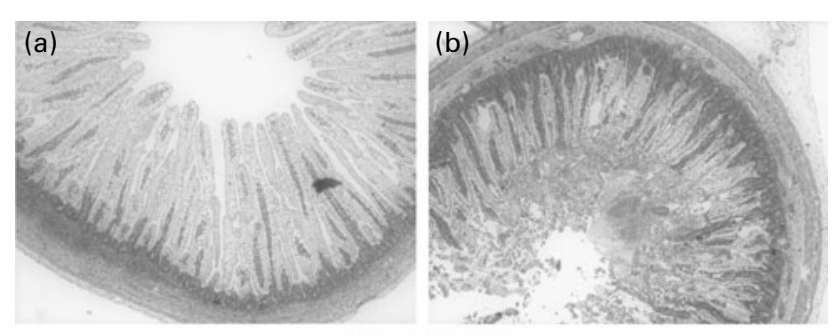

(c)

was significantly reduced in the OPN group $(2 \cdot 5$ (SEM 0.3$)$ v. 3.7 ( formula groups showed high NEC incidence (83-100\%), while only $40 \%(2 / 5)$ of the pigs in the colostrum group developed NEC (Table 2). Only mild signs of NEC (grades 2 and 3) were observed in the colon and distal SI of two pigs fed colostrum. Hence, the NEC severity score of the colostrum group was significantly lower $(P<0.0001)$ than all three formula-fed groups, which did not differ. In both the experiments, there were no significant differences in organ weights, intestinal dimensions and mucosal proportions among treated groups and their respective control groups (data not shown).

Intestinal mucosal morphology and enzyme activities. Microscopic examination of haematoxylin and eosin-stained sections of the distal SI revealed obvious pathological differences between pigs macroscopically diagnosed with NEC compared to those pigs diagnosed as healthy. The NEC pigs had extensive bleeding and mucosal atrophy, and in very severe cases (NEC severity score of 5-6), complete loss and disintegration of the mucosa, submucosa and muscularis layers were observed (Fig. 2). Measurement of villus height showed no significant differences between the OPN and control groups in Expt 1 or among the four groups in Expt 2 (Table 2).

There was no statistical difference in the activity of any of the measured enzymes between OPN and control pigs (Expt 1) and among the Gang, SL and control formula groups in Expt 2 (Table 2). Pigs fed bovine colostrum had significantly higher activities of all measured enzymes relative to the control formula group. Compared with the
Gang and SL groups, all the enzyme activities except for sucrase and maltase were also significantly improved with bovine colostrum, most pronounced for lactase (approximately a twofold activity increase, Expt 2, Table 2).

\section{Expt 3: in vitro immunomodulatory activity of milk fractions}

For all four measured cytokines (IL-10, IL-12, IL-6 and $\mathrm{TNF}-\alpha$ ), there were no significant differences between the bacteria-stimulated DC and the DC co-stimulated with bacteria and $0 \cdot 1,0 \cdot 5,1$ and $5 \mu \mathrm{g} / \mathrm{ml}$ of OPN (data not shown). The Gang-enriched milk product added to DC alone did not induce a cytokine response from the cells (Fig. 3(a) and (b)). High doses of the Gang-enriched milk product co-incubated with DC and bacteria resulted in reduced production of IL-10 and IL-12, with strongest inhibition of E. coli Nissle-induced cytokine production (Fig. 3(a) and (b)). There were no effects of Gang on the TNF- $\alpha$ and IL-6 responses (data not shown). When the SL-enriched milk product was added to the DC alone, it induced production of all four cytokines (Fig. 3(c) and (d)) Upon co-stimulation with bacteria, no effect on cytokine production of the SL-enriched milk product was observed for doses lower than $250 \mu \mathrm{g} / \mathrm{ml}$. Conversely, higher concentrations resulted in a general increase in cytokine production independently of bacterial stimulation (Fig. 3(c) and (d), IL-6 and TNF- $\alpha$ data are not shown).

Defatted colostrum was associated with high mortality of DC, and only $0.9 \%$ colostrum was tolerated by the cells resulting in similar effects as for low concentrations of whey (data are not shown). Therefore, cytokine production was measured after co-incubation with $10 \mu \mathrm{g} / \mathrm{ml}$ C. perfringens NEC A20 or E. coli Nissle 1917 and 8.3, 2.8 and $0.9 \%(\mathrm{v} / \mathrm{v})$ of bovine colostrum whey, which was not lethal to the DC. Colostrum whey alone induced some production of all four cytokines except IL-12 (Fig. 4). The whey fraction caused a bacteria-dependent modulation of the cytokine response from the stimulated DC. For E. coli-stimulated cells, whey caused a dosedependent decrease in production of all cytokines (Fig. 4(a)-(d), $P<0.01$ and 0.001), while inhibiting effects on cytokine production were modest after $C$. perfringens stimulation. Production of IL-10 was induced with whey only after $C$. perfringens stimulation (Fig. 4(a), $P<0.05$ and $0 \cdot 01$ ).

\section{Discussion}

The results show that enteral feeding with bovine colostrum reduces NEC lesions in preterm pigs, relative to formula, as shown previously for porcine colostrum $^{(29,33-35,40,42,43)}$. It now remains to be investigated whether bovine colostrum, or fractions thereof, might also prevent gut inflammation and NEC in preterm infants lacking access to mother's milk. Formulas enriched with 


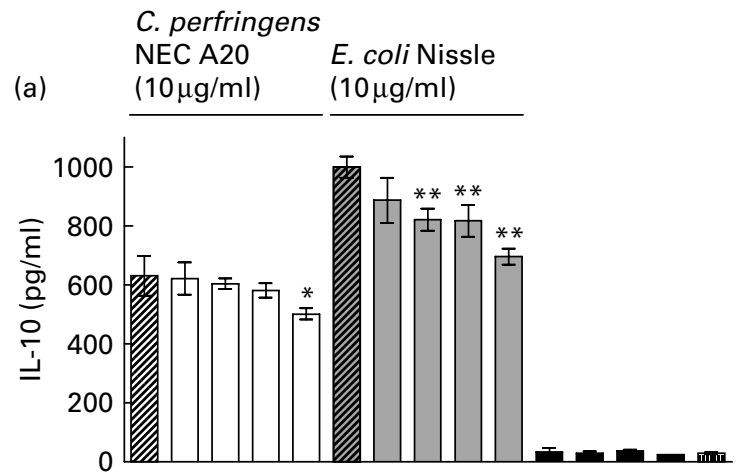

(b)

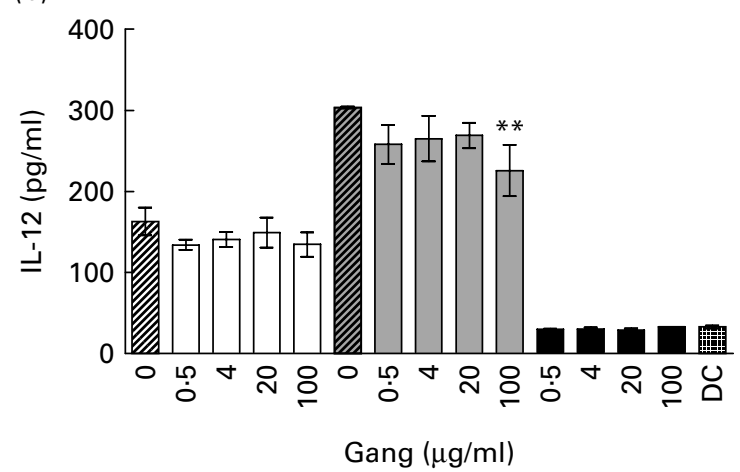

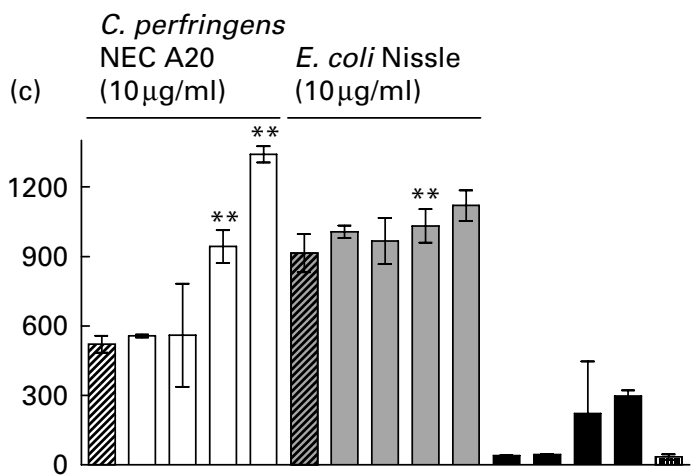

(d)

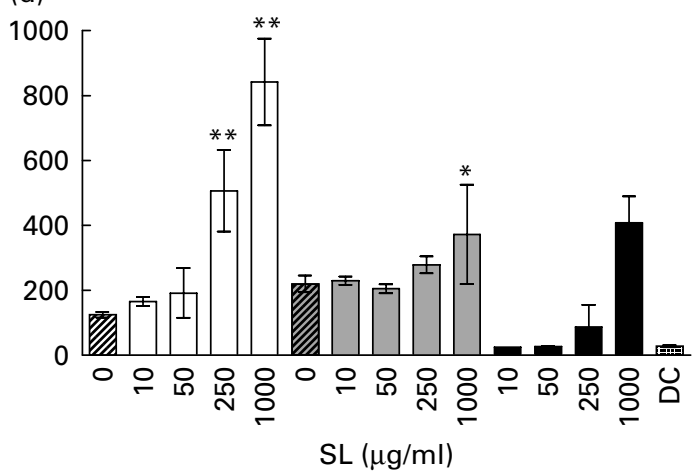

Fig. 3. IL-10 ((a) and (c)) and IL-12 ((b) and (d)) expression in dendritic cells (DC) co-incubated with $10 \mu \mathrm{g} / \mathrm{ml}$ Clostridium perfringens NEC A20 or Escherichia coli Nissle 1917 and different concentrations of sialic acids (SL) and gangliosides (Gang). The results are means and standard deviations of three replicates from one experiment, representative of two separate experiments. Values are compared to their respective controls without SL and Gang (striped bars). Mean values were significantly different: ${ }^{\star} P<0.05,{ }^{*} P<0.01$.

Gang or SL failed to improve resistance in pigs, while OPN supplementation was associated with a modest decrease in NEC without any consistent effects on gut structural and functional indices. The potential immune-modulating activity of bovine colostrum was supported by the effects of colostrum whey on DC stimulated in vitro with either E. coli Nissle 1917 (a commensal probiotic bacteria) or C. perfringens NEC A20 (an opportunistic NEC pathogen). In contrast, the enriched milk fractions showed no consistent effects, except that high concentration of Gang reduced the IL-10 and IL-12 production in bacteria-stimulated DC.

In vivo, bovine colostrum improved several gut functional indices (galactose and mannitol absorption, dipeptidyl-peptidase IV, aminopeptidase A, aminopeptidase $\mathrm{N}$ and lactase activities), while the effects of enriched formulas were limited or absent, at least for the doses chosen in the present study. The activities of six intestinal brush-border enzymes were elevated in pigs fed bovine colostrum, relative to formula, confirming previous findings with porcine colostrum ${ }^{(29,33,34,40)}$. Digestive enzyme activities and nutrient absorptive capacity are sensitive parameters to detect digestive dysfunction, and they often correlate well with gut integrity and degree of inflammation $^{(44)}$. In the present study, both the galactose and mannitol absorptions were reduced $1 \mathrm{~d}$ after initiation of enteral formula feeding. Other recent studies have indicated that this formula-induced decrease in nutrient absorptive capacity may take place a few hours after the start of feeding ${ }^{(45)}$, coinciding with a very rapid formulainduced increase in tissue inflammatory markers such as IL- $1 \beta^{(31)}$, but before structural changes. In the present study, colostrum did not improve mucosal function by stimulating structural integrity, since we found no significant treatment differences for villus height, mucosa proportion or intestinal weight in the two experiments. Mean villus height in the bovine colostrum group was relatively low $(472 \mu \mathrm{m})$ compared with preterm pigs fed porcine or bovine colostrum starting from the time of birth, without a TPN period (approximately $600 \mu \mathrm{m})^{(30,34)}$. Colostrum was not fully protective, as two of the five colostrum pigs showed mild NEC lesions in the distal intestine and colon. In agreement with our earlier observations $^{(29)}$, a few days of TPN after birth may predispose preterm pigs to moderate inflammatory lesions, even when a near-optimal diet (porcine or bovine colostrum and OPN-enriched formula) is used as an enteral diet after TPN. Furthermore, we have also previously observed that minimal enteral doses of bovine colostrum during TPN followed by full enteral nutrition with bovine colostrum fully protect against $\mathrm{NEC}^{(46)}$. These previous findings with bovine colostrum indicate that it is only fully protective against NEC in the preterm pig model if given immediately after birth as it seems to counteract the detrimental effects of initial TPN. 

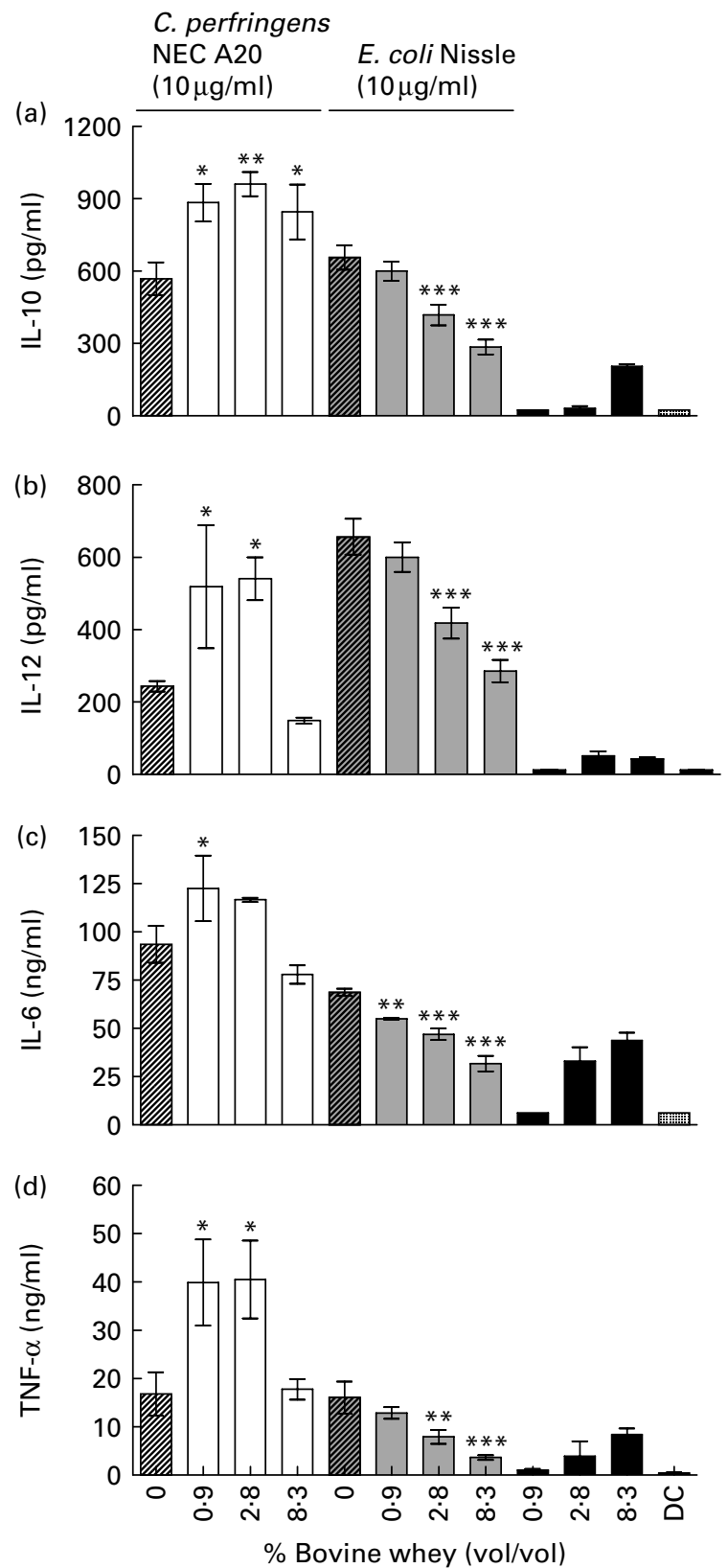

Fig. 4. IL-10 (a), IL-12 (b), IL-6 (c) and TNF- $\alpha$ (d) expression in dendritic cells (DC) co-incubated with $10 \mu \mathrm{g} / \mathrm{ml}$ Clostridium perfringens NEC A20 or Escherichia coli Nissle 1917 and different concentrations of a whey fraction from bovine colostrum. The results are means and standard deviations of three replicates from one experiment, representative of two separate experiments. Values are compared to their respective controls without whey (striped bars). Mean values were significantly different: ${ }^{*} P<0.05$, ${ }^{\star *} P<0.01$, ${ }^{* * *} P<0.001$.

In vitro, bovine colostrum whey caused a bacteriadependent modulation of cytokine responses from stimulated DC. In recent studies with the DC model, we have found similar modulatory effects of porcine and human whey, indicating conserved immunomodulatory effects between different species (HK Møller, unpublished results). In contrast, the enriched milk fractions showed no consistent effects, except high concentration of Gang, which reduced the IL-10 and IL-12 production in bacteria-stimulated DC.

In vitro, it has recently been shown that a bovine colostrum protein concentrate is able to modulate cytokine production from lipopolysaccharide-stimulated human peripheral blood mononuclear cells by increasing release of IL-2 while suppressing early production of TNF, IL-6 and IL- ${ }^{(47)}$. The same study found an increase in IL-10 and IL-2 production and inhibition of interferon- $\gamma$ and TNF, when cells were stimulated with phytohaemagglutinin in the presence of colostrum ${ }^{(47)}$. Another study found that bovine colostrum was able to modulate interferon- $\gamma$ production in stimulated human peripheral blood mononuclear cells depending on the strength and type of antigenic stimulation ${ }^{(48)}$. Our findings using murine DC extend these properties to cell types other than peripheral blood mononuclear cells. As the present results were obtained using the whey fraction of colostrum, they, together with the Shing et al. study ${ }^{(47)}$, indicate that the immunomodulatory properties may be due to bioactive whey proteins. Previously, we have found immunosuppressive activity of sialylated milk proteins on the DC cytokine response to lipopolysaccharide, which again supports the notion that the proteins of colostrum play an important role in preventing inflammation ${ }^{(28)}$. It must also be noted that the in vitro data indicate a stimulus dependency of the effects of colostrum, which may be an important regulatory mechanism behind the protective effects of colostrum against inflammation in vivo.

The present study is the first to test luminal effects of OPN in a porcine model of gut immaturity and inflammation. Based on the immune-modulating effects of OPN documented by others under other conditions $^{(4,5,8,11,13,14,49-51)}$, we hypothesised that milkderived OPN played a role in the control of inflammatory responses in neonates. OPN exhibits pro-survival and anti-apoptotic properties, and thus aids cell survival ${ }^{(50)}$, wound healing and matrix formation ${ }^{(11,12,52)}$, supporting the observed tendency to positive clinical effects in the present study, where OPN may positively affect rebuilding of necrotic mucosa and tissue or through anti-apoptotic signals promoting epithelial cell survival. Further studies on OPN concentrations and mode of administration (enteral $v$. parenteral) are warranted. Although OPN significantly reduced NEC severity, the clinical effects of OPN on gut function parameters using the present treatment regimen were minimal. It is possible that OPN effects are developmentally dependent and that the gut-associated lymphoid tissue is too immature in preterm pigs to be able to fully respond to OPN.

Developmental immaturity may also contribute to the lacking effects of SL- and Gang-enriched formulas. Furthermore, the concentrations of these bioactives in the formulas may have been too low to provide beneficial effects in vivo. The finding that SL only affected DC 
function at very high concentrations could indicate that a DC-stimulating contaminant, such as lipopolysaccharide, may have been present in the enriched formula. Different structures of Gang have been shown to exhibit immunosuppressive effects on various immunocompetent cell types in vitro, and these effects seem to be dependent on the Gang structure and type of immune cell affected $^{(17,18,24)}$. Clinically, a Gang-enriched formula has been found to stimulate the development of a beneficial gut microbiota in preterm infants, relative to control formula ${ }^{(19)}$. Milk Gang and conjugated sialylated carbohydrates (glycoproteins and glycolipids) may also serve as false receptor analogues for bacterial adhesins, and thus be able to modify the intestinal microbiota in the neonate serving as a beneficial prebiotic.

As in human milk, the predominant Gang of bovine milk are disialganglioside 3 (GD3) and monosialganglioside 3 (GM3), but in general, human milk contains higher amounts of Gang than bovine $\operatorname{milk}^{(7,19)}$. Hence, the Gang-rich milk product employed in the present study most likely contains GD3 and GM3, and concentrations approached those in human milk. We observed a dosedependent suppression of E. coli Nissle 1917-induced IL-10 and IL-12 cytokine production, probably due to the effects of GD3 and GM3. This slight immunosuppressive effect of the Gang milk product on the DC cytokine response confirms our previous findings with pure Gang $^{(18)}$. In conclusion, bovine colostrum was superior to milk formulas enriched with milk OPN, Gang or SL in protecting against NEC in preterm piglets. OPN tended to reduce NEC as indicated by a clearly reduced severity score but without any consistent improvement of structural and functional indices. The modest effect of milk bioactives may be explained by the immature state of the gut immune system in preterm pigs. Equally well, the effects of the enriched formulas may just be minimal compared with that of the synergistic actions of many bioactives present in intact bovine colostrum. These synergistic actions of colostral bioactives appear to include inhibitory effects on bacteria-stimulated DC cytokine release.

\section{Acknowledgements}

We gratefully acknowledge the technical assistance from Elin Skytte, Bente Synnestvedt and Anni Mehlsen. The work was supported by the FOSU-DSF programme by the Danish Research Councils, the Technical University of Denmark and Arla Foods Ingredients. One of the co-authors, A. S. K., is employed by Arla. Although she took part in the design of the studies, and initial interpretation of the results, final responsibility for the manuscript is with the remaining authors who had no potential conflicts of interest. P. T. S., T. T., L. N. F., H. F. and A. S. K. designed the research; H. K. M., P. T. S. and T. T. conducted the research; A. S. K. provided essential reagents; H. K. M. and T. T. analysed data; H. K. M., T. T., L. N. F., H. F. and P. T. S. wrote the paper. H. K. M. had primary responsibility for the final content. All authors read and approved the final manuscript.

\section{References}

1. Kelleher SL \& Lönnerdal B (2001) Immunological activities associated with milk. Adv Nutr Res 10, 39-65.

2. Field CJ (2005) The immunological components of human milk and their effect on immune development in infants. J Nutr 135, 1-4.

3. Edwards CA \& Parrett AM (2002) Intestinal flora during the first months of life: new perspectives. Br J Nutr 88, S11-S18.

4. Ashkar S, Weber GF, Panoutsakopoulou V, et al. (2000) Eta-1 (osteopontin): an early component of type-1 (cell-mediated) immunity. Science 287, 860-864.

5. Begum MD, Umemura M, Kon S, et al. (2007) Suppression of the bacterial antigen-specific $\mathrm{T}$ cell response and the dendritic cell migration to the lymph nodes by osteopontin. Microbiol Immunol 51, 135-147.

6. Boehm G, Lidestri M, Casetta P, et al. (2002) Supplementation of a bovine milk formula with an oligosaccharide mixture increases counts of faecal bifidobacteria in preterm infants. Arch Dis Child 86, 178-181.

7. Martin MJ, Martin-Sosa S, Alonso JM, et al. (2003) Enterotoxigenic Escherichia coli strains bind bovine milk gangliosides in a ceramide-dependent process. Lipids 38, 761-768.

8. Renkl AC, Wussler J, Ahrens T, et al. (2005) Osteopontin functionally activates dendritic cells and induces their differentiation toward a Th1-polarizing phenotype. Blood 106, 946-955.

9. Varki A (2007) Glycan-based interactions involving vertebrate sialic-acid-recognizing proteins. Nature $\mathbf{4 4 6}$, 1023-1029.

10. Wang B \& Brand-Miller J (2003) The role and potential of sialic acid in human nutrition. Eur J Clin Nutr 57, 1351-1369.

11. Denhardt DT, Noda M, O'Regan AW, et al. (2001) Osteopontin as a means to cope with environmental insults: regulation of inflammation, tissue remodeling, and cell survival. J Clin Invest 107, 1055-1061.

12. Sodek J, Ganss B \& McKee MD (2000) Osteopontin. Crit Rev Oral Biol Med 11, 279-303.

13. Sato T, Nakai T, Tamura N, et al. (2005) Osteopontin/Eta-1 upregulated in Crohn's disease regulates the Th1 immune response. Gut 54, 1254-1262.

14. Sodek J, Batista Da Silva AP \& Zohar R (2006) Osteopontin and mucosal protection. $J$ Dent Res 85, 404-415.

15. Agnholt J, Kelsen J, Schack L, et al. (2007) Osteopontin, a protein with cytokine-like properties, is associated with inflammation in Crohn's disease. Scan J Immunol 65, 453-460.

16. Scatena M, Liaw L \& Giachelli CM (2007) Osteopontin: a multifunctional molecule regulating chronic inflammation and vascular disease. Arterioscler Thromb Vasc Biol 27, 2302-2309.

17. Bergelson LD (1995) Serum gangliosides as endogenous immunomodulators. Immunol Today 16, 483-486.

18. Bronnum H, Seested T, Hellgren LI, et al. (2005) Milk-derived GM(3) and GD(3) differentially inhibit dendritic cell maturation and effector functionalities. Scand J Immunol 61, 551-557.

19. Rueda R, Maldonado J, Narbona E, et al. (1998) Neonatal dietary gangliosides. Early Hum Dev 53, S135-S147.

20. Takamizawa K, Iwamori M, Mutai M, et al. (1986) Selective changes in gangliosides of human-milk during lactation a molecular indicator for the period of lactation. Biochim Biophys Acta 879, 73-77. 
21. Martin-Sosa S, Martin MJ, Garcia-Pardo LA, et al. (2003) Sialyloligosaccharides in human and bovine milk and in infant formulas: variations with the progression of lactation. J Dairy Sci 86, 52-59.

22. Pan XL \& Izumi T (2000) Variation of the ganglioside compositions of human milk, cow's milk and infant formulas. Early Hum Dev 57, 25-31.

23. Rueda R (2007) The role of dietary gangliosides on immunity and the prevention of infection. Br J Nutr 98, S68-S73.

24. Ladisch S, Becker H \& Ulsh L (1992) Immunosuppression by human gangliosides: I. Relationship of carbohydrate structure to the inhibition of $\mathrm{T}$ cell responses. Biochim Biophys Acta Lipids Lipid Metabol 1125, 180-188.

25. Varki A (2008) Sialic acids in human health and disease. Trends Mol Med 14, 351-360.

26. Laegreid A, Otnaess ABK \& Fuglesang J (1986) Human and bovine-milk - comparison of ganglioside composition and enterotoxin-inhibitory activity. Pediatr Res 20, 416-421.

27. Christensen HR, Frokiar H \& Pestka JJ (2002) Lactobacilli differentially modulate expression of cytokines and maturation surface markers in murine dendritic cells. J Immunol 168, 171-178.

28. Mikkelsen TL, Bakman S, Sorensen ES, et al. (2005) Sialic acid-containing milk proteins show differential immunomodulatory activities independent of sialic acid. J Agric Food Chem 53, 7673-7680.

29. Bjornvad CR, Thymann T, Deutz NE, et al. (2008) Enteral feeding induces diet-dependent mucosal dysfunction, bacterial proliferation, and necrotizing enterocolitis in preterm pigs on parenteral nutrition. Am J Physiol Gastrointest Liver Physiol 295, G1092-G1103.

30. Sangild PT, Siggers RH, Schmidt M, et al. (2006) Diet- and colonization-dependent intestinal dysfunction predisposes to necrotizing enterocolitis in preterm pigs. Gastroenterology 130, 1776-1792.

31. Van Haver ER, Oste M, Thymann T, et al. (2008) Enteral feeding reduces endothelial nitric oxide synthase in the caudal intestinal microvasculature of preterm piglets. Pediatr Res 63, 137-142.

32. De la Cochetiere MF, Piloquet H, Des Robert C, et al. (2004) Early intestinal bacterial colonization and necrotizing enterocolitis in premature infants: the putative role of Clostridium. Pediatr Res 56, 366-370.

33. Sangild PT, Bjornvad CR, Petersen YM, et al. (2002) Spontaneous NEC-like symptoms in formula-fed premature pigs. Gastroenterology 122, A278.

34. Siggers RH, Siggers J, Boye M, et al. (2008) Early administration of probiotics alters bacterial colonization and limits diet-induced gut dysfunction and severity of necrotizing enterocolitis in preterm pigs. J Nutr 138, 1437-1444.

35. Siggers RH, Thymann T, Jensen BB, et al. (2008) Elective cesarean delivery affects gut maturation and delays microbial colonization but does not increase necrotizing enterocolitis in preterm pigs. Am J Physiol Regul Integr Comp Physiol 294, R929-R938.

36. Schack L, Lange A, Kelsen J, et al. (2009) Considerable variation in the concentration of osteopontin in human milk, bovine milk, and infant formulas. J Dairy Sci 92, 5378-5385.
37. Gill HS, Doull F, Rutherfurd KJ, et al. (2000) Immunoregulatory peptides in bovine milk. Br J Nutr 84, 111-117.

38. Varki NM \& Varki A (2007) Diversity in cell surface sialic acid presentations: implications for biology and disease. Lab Invest 87, 851-857.

39. Kurz G \& Wallenfels K (1974) D-Galactose UV assay with galactose dehydrogenase. In Methods of Enzymatic Analysis, 2nd ed., pp. 1279-1282 [HU Bergmeyer, editor]. New York: Academic Press.

40. Thymann T, Burrin DG, Tappenden KA, et al. (2006) Formula-feeding reduces lactose digestive capacity in neonatal pigs. Br J Nutr 95, 1075-1081.

41. Sangild PT, Sjostrom H, Noren O, et al. (1995) The prenatal development and glucocorticoid control of brush-border hydrolases in the pig small-intestine. Pediatr Res 37, 207-212.

42. Oste M, Van Ginneken CJ, Van Haver ER, et al. (2005) The intestinal trophic response to enteral food is reduced in parenterally fed preterm pigs and is associated with more nitrergic neurons. J Nutr 135, 2657-2663.

43. Sangild PT, Petersen YM, Schmidt M, et al. (2002) Preterm birth affects the intestinal response to parenteral and enteral nutrition in newborn pigs. J Nutr 132, 2673-2681.

44. Buddington RK, Bering SB, Thymann T, et al. (2008) Aldohexose malabsorption in preterm pigs is directly related to the severity of necrotizing enterocolitis. Pediatr Res 63, 382-387.

45. Thymann T, Moller HK, Stoll B, et al. (2009) Carbohydrate maldigestion induces necrotizing enterocolitis in preterm pigs. Am J Physiol Gastrointest Liver Physiol 297, G1115-G1125.

46. Cilieborg MS, Boye M, Thymann T, et al. (2010) Diet-dependent effects of minimal enteral nutrition on intestinal function and necrotizing enterocolitis in preterm pigs. JPEN J Parenter Enteral Nutr (In the Press).

47. Shing CM, Peake JM, Suzuki K, et al. (2009) Bovine colostrum modulates cytokine production in human peripheral blood mononuclear cells stimulated with lipopolysaccharide and phytohemagglutinin. J Interferon Cytokine Res 29, $37-44$.

48. Biswas P, Vecchi A, Mantegani P, et al. (2007) Immunomodulatory effects of bovine colostrum in human peripheral blood mononuclear cells. New Microbiol 30, 447-454.

49. Giachelli CM, Lombardi D, Johnson RJ, et al. (1998) Evidence for a role of osteopontin in macrophage infiltration in response to pathological stimuli in vivo. Am J Pathol 152, $353-358$

50. Khan SA, Lopez-Chua CA, Zhang J, et al. (2002) Soluble osteopontin inhibits apoptosis of adherent endothelial cells deprived of growth factors. J Cell Biochem 85, 728-736.

51. Xanthou G, Alissafi T, Semitekolou M, et al. (2007) Osteopontin has a crucial role in allergic airway disease through regulation of dendritic cell subsets. Nat Med 13, 570-578.

52. Da Silva APB, Pollett A, Rittling SR, et al. (2006) Exacerbated tissue destruction in DSS-induced acute colitis of OPN-null mice is associated with downregulation of TNF-alpha expression and non-programmed cell death. $J$ Cell Physiol 208, 629-639. 